\title{
Primary Pulmonary Mucosa-Associated Lymphoid Tissue Lymphoma Accompanied by a Hydatid Cyst
}

\author{
Cemil Göya MD, Cihad Hamidi MD, Mehmet Güli Çetinçakmak MD, \\ Salih Hattapoğlu MD, and Memik Teke MD
}

\section{Introduction}

Pulmonary lymphomas are categorized into 4 groups: primary pulmonary involvement, recurrent or secondary pulmonary lymphoma, post-transplantation lymphoproliferative lymphomas, and lymphomas related to the acquired immune deficiency syndrome. ${ }^{1-3}$

Primary pulmonary lymphoma (PPL) is a rare entity composing $\sim 0.5-1.0 \%$ of all pulmonary malignancies and $<1.0 \%$ of all lymphomas. PPL affects both lobes of the lungs (parenchyma or bronchial) and is described as a clonal lymphoid proliferative disease. In these patients, no mediastinal adenopathy or extrapulmonary involvement occurs. However, extrathoracic involvement is expected during the first 3 months of the follow-up period. ${ }^{4,5}$ PPL is most commonly known as a non-Hodgkin lymphoma and has been divided into 2 World Health Organization groups: mucosa-associated lymphoid tissue (MALT) and nonMALT. MALT defines the lymphoid tissue located beneath the epithelial tissue that is associated with mucosal immunity. ${ }^{6-9}$ When lymphoma occurs in the lungs, factors such as smoking, autoimmune deficiencies, and infectious diseases are considered to correlate with the chronic antigenic stimulation of the disease..$^{1,4-6,10,11}$

Primary pulmonary MALT lymphoma is most commonly seen between the 5th and 7th decades of life and rarely observed before $30 \mathrm{y}$ of age. The disease occurrence has been thought to be similar in both sexes, but recent literature has reported a higher incidence in female patients. ${ }^{7}$

The symptoms of pulmonary lymphomas include single or multiple nodules, masses, infiltrates, and radiological

The authors are affiliated with the Department of Radiology, Medical School, Dicle University, Diyarbakır, Turkey.

The authors have disclosed no conflicts of interest.

Correspondence: Cemil Göya MD, Department of Radiology, Medical School, Dicle University, Yenişehir, 21280 Diyarbakır, Turkey. E-mail: cegoya1@yahoo.com.

DOI: $10.4187 /$ respcare. 03113 findings such as consolidation. Primary pulmonary MALT is a very rare disease and, in our case, was accompanied by a hydatid cyst, which is discussed in this report.

\section{Case Summary}

A 72-y-old male patient was admitted to the clinic and complained of tiredness and progressive dyspnea for the past 2 months. There was no past medical history of weight loss, alcohol use, or cigarettes. Physical examination of the subject determined that he was weak, with a blood pressure measurement of $110 / 70 \mathrm{~mm} \mathrm{Hg}$ and a heart rate of 90 beats/min.

Further analysis confirmed inspiratory crackles at the bottom zones of both hemithoraces. Other examination findings were normal. However, laboratory findings revealed a red blood cell count of 5 million cells $/ \mu \mathrm{L}$, hemoglobin levels of $10.9 \mathrm{~g} / \mathrm{dL}$, and a hematocrit of $34.9 \%$. The white blood cell count was 4,500 cells $/ \mu \mathrm{L}$, and the erythrocyte sedimentation rate was $56 \mathrm{~mm} / \mathrm{h}$. After surgery, recurring hydatid cyst lesions were observed by abdominal tomography, and the serology for a hydatid cyst was positive. An indirect hemagglutination test was used for detection of specific anti-Echinococcus granulosus antibody.

The first chest computed tomography (CT) showed a ground-glass opacity of low attenuation areas located at the perihilar regions of both lung lobes. In addition, consolidation, peribronchial thickness, and nodular lesions were also observed (Fig. 1). Moreover, a hydatid cyst cavity, which was treated surgically, was present in the left lung as observed by thoracic tomography (Fig. 2). A bone marrow biopsy was also performed. Histologically, there were 3 nodular and 2 patchy infiltration foci of small lymphocytes. The lymphocytes were CD20-positive and CD5-negative. The morphological and clinical findings, which included bone marrow involvement, confirmed the significant diagnostic evidence for MALT lymphoma infiltration. After treatment, the pulmonary symptoms regressed.

Transbronchial biopsy revealed infiltration of atypical lymphoid B cells. Histologically, the biopsy showed dif- 


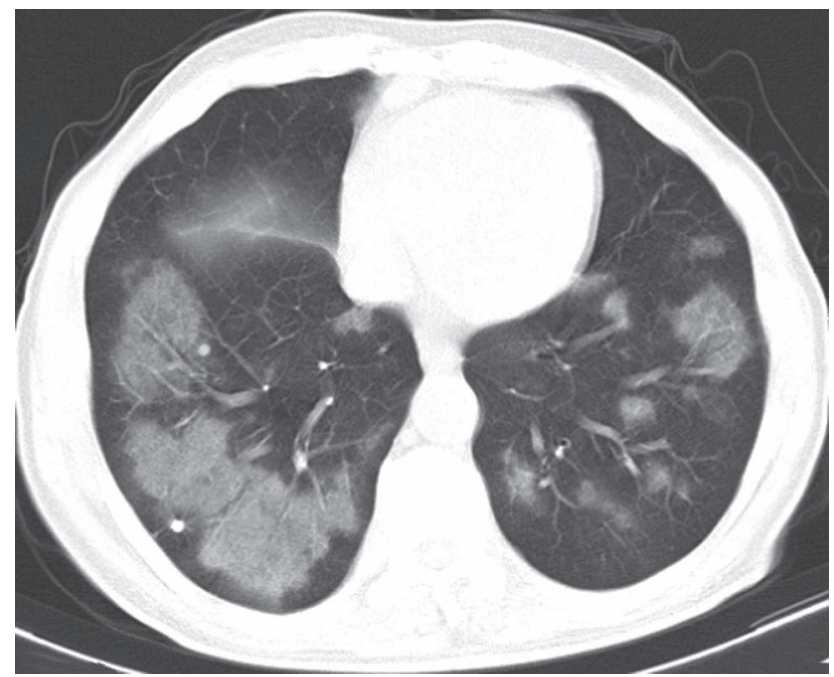

Fig. 1. Axial computed tomogram shows disperse consolidated areas and nodules in the lower lobes of both lungs.

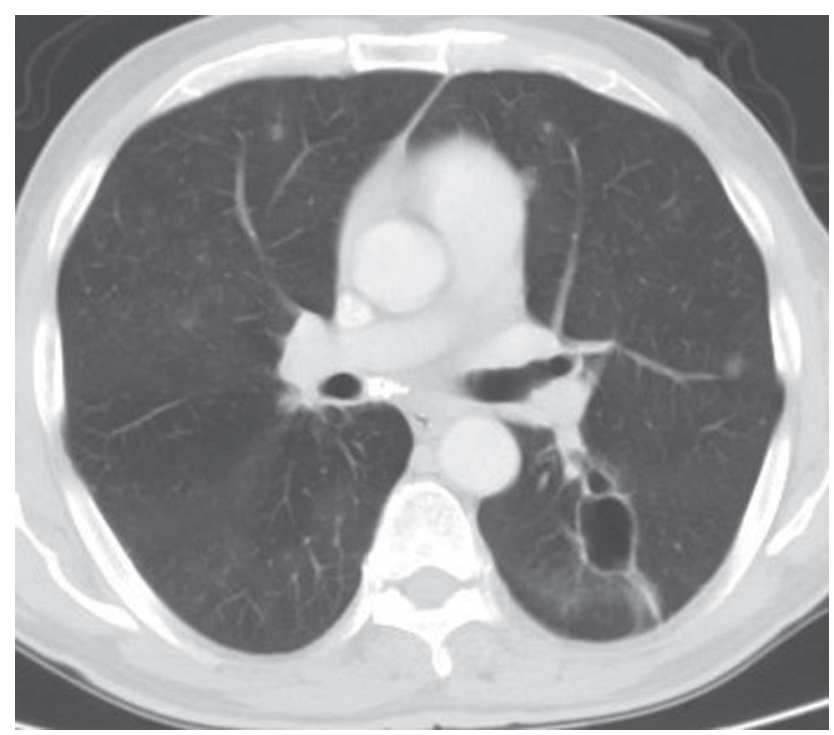

Fig. 2. Axial computed tomogram shows cystic sequela change due to hydatid cyst surgery in the superior segment of the lower lobe of the left lung.

fuse infiltration of small lymphocytes with focal plasma cell differentiation. In addition, an immunohistochemical study was performed. Small lymphocytes were positive for CD20 but negative for CD5, CD23, Bcl-1, and CD45RO. CD38-positive plasma cells were present in small numbers ( $5 \%$ of all lymphoid cells). The lymphoid cells were largely positive for the $\kappa$ light chain, and only a few cells were positive for the $\lambda$ light chain $(\kappa / \lambda$ ratio of $4-10: 1)$ (Figs. 3 and $4, \mathrm{~A}$ and $\mathrm{B}$ ). These immunohistochemical data, together with histological findings, confirmed that the existing lesion was a low-grade MALT lymphoma.

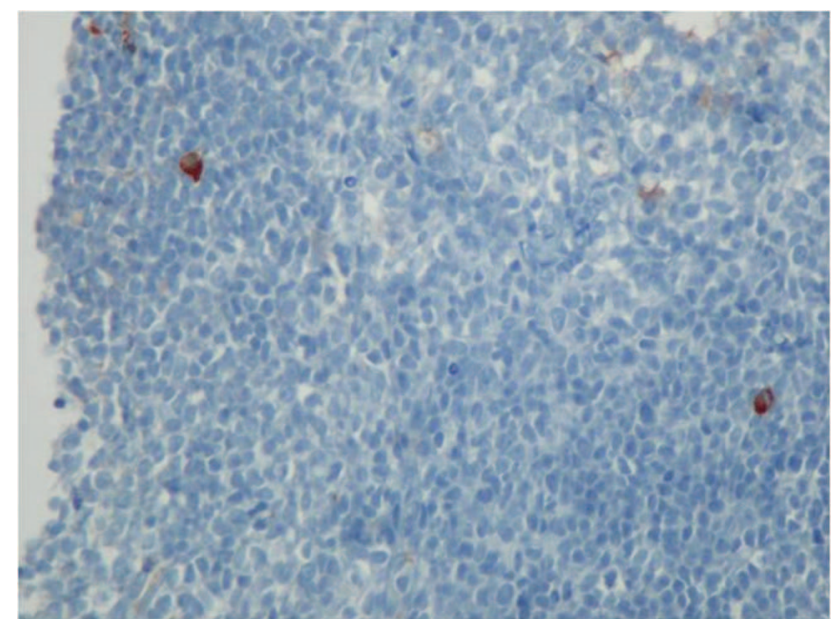

Fig. 3. The lymphocytes stained positively for the $\kappa$ light chain, shown at $\times 200$ magnification.

Furthermore, a bone marrow biopsy was performed and showed no neoplastic infiltration. Upper gastrointestinal endoscopy did not reveal areas of metaplasia. No trace of Helicobacter pylori was observed. The absence of lymphadenopathy or other signs of extrathoracic disease in the abdominal CT suggested that the lymphoma was a primary type with exclusive pulmonary involvement.

The subject received 5 cycles of chemotherapy that included cyclophosphamide, vincristine, and prednisone over a 3-month period, with complete remission of the symptoms. He underwent clinical and radiological follow-up for a 3-y period with CT, and no changes were detected.

\section{Discussion}

Pulmonary MALT lymphoma is a rare disease. In addition, co-occurrence with a hydatid cyst is a low-probability comorbidity. Pulmonary lesions are confined mostly to the lungs, and progression is generally slow. The time between the initial clinical findings and radiological manifestations is from 5 months to 8 y. ${ }^{1,6}$

Nonspecific symptoms such as cough, mild dyspnea, chest pain, and rarely hemoptysis may occur. Pulmonary auscultation symptoms may be seen in occasional cases. ${ }^{1}$ In our subject, the existing symptoms included tiredness, progressive dyspnea, nonspecific laboratory findings, and consolidation on chest radiography.

The most frequent radiological findings in PPL are unilateral or bilateral pulmonary infiltrations or nodules and peribronchial thickening. ${ }^{2-4,6}$ Radiography shows localized alveolar opacities with irregular boundaries and air bronchograms in half of PPL patients. ${ }^{1,9} \mathrm{CT}$ is more sensitive, and consolidations are observed as ground-glass opacities and peribronchial thickness. These symptoms are mostly bilateral and multiple. ${ }^{3,9,10}$ 


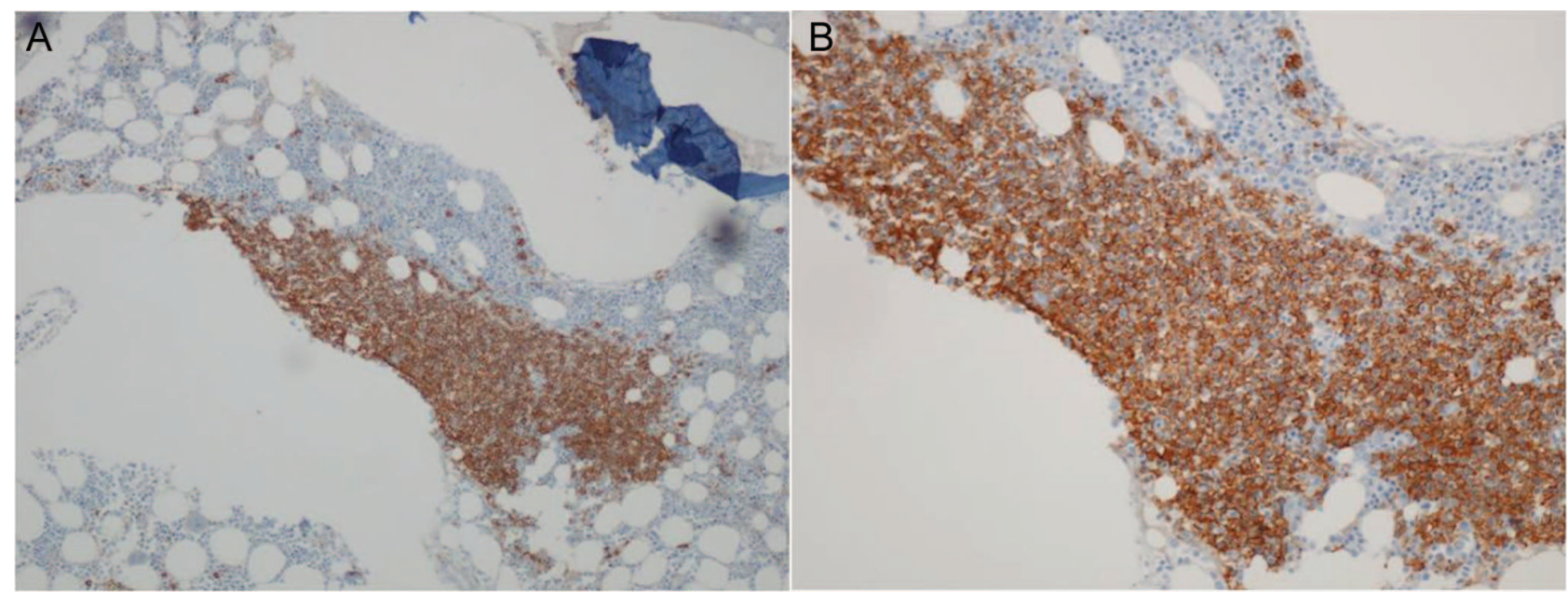

Fig. 4. CD20 expression in small lymphocytes, shown at $\times 200$ magnification $(A)$ and at $\times 400$ magnification (B).

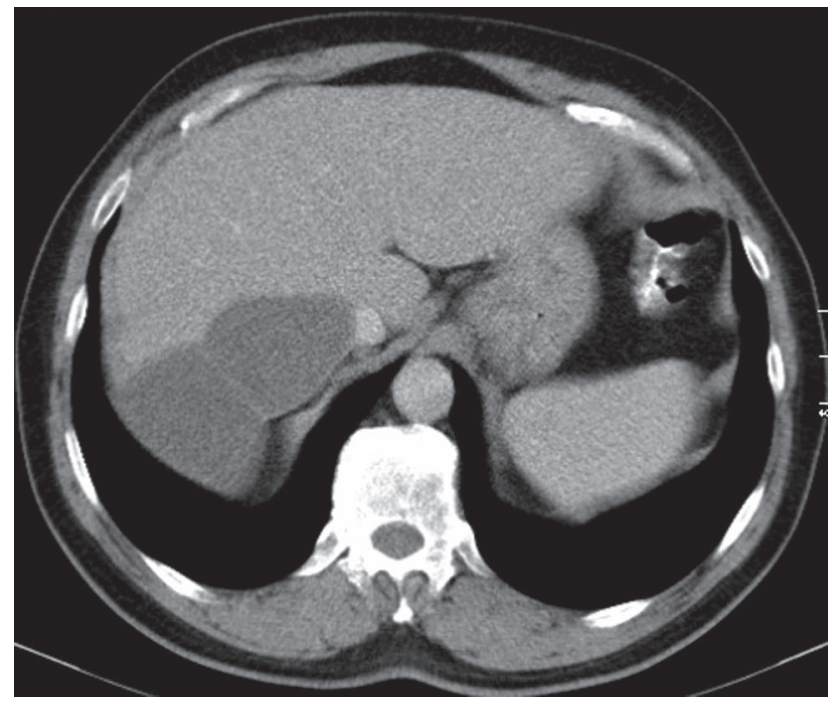

Fig. 5. Hydatid cyst lesions in the posterior segment of the right lobe of the liver.

Diagnostic criteria for PPL are not certain and necessitate histological, immunohistochemical, and molecular biological findings. To evaluate the dispersion, upper and lower gastrointestinal endoscopy and bone marrow biopsy are essential. ${ }^{4}$

In bronchoalveolar lavage, increased cellularity is often seen with a high proportion of lymphocytes. Histological diagnosis is made with bronchial or transbronchial biopsy and transthoracic needle aspiration biopsy. ${ }^{7}$

It has been proposed that mutations that occur during B cell response to helicobacterium antigens in $H$. pylori infections may be involved in the pathogenesis of gastrointestinal tract MALT lymphomas. In addition, chronic antigen stimulation may play a role in MALT lymphomas in other regions. The specific antigens that take part in pul- monary MALT lymphomas have not been determined. ${ }^{11}$ The case report that has been presented here draws attention to patients with a previous hydatid cyst, an old hydatid cyst cavity, and multiple active hydatid cyst lesions in the liver (Fig. 5).

\section{Teaching Points}

- Pulmonary MALT lymphoma is a rare disease, and cooccurrence with a hydatid cyst is a low-probability comorbidity.

- This co-occurrence of PPL and a hydatid cyst has not been reported in the literature.

- The most frequent radiological findings in PPL are mostly bilateral and multiple pulmonary infiltrations or nodules and peribronchial thickening.

- $\mathrm{CT}$ is more sensitive, and consolidations are observed as ground-glass opacities and peribronchial thickness. Other non-pulmonary pathology is the same as that for a hydatid cyst.

- Primary pulmonary non-Hodgkin lymphoma is a disease that progresses slowly and is prognostic in nature and responsive to treatment.

\section{REFERENCES}

1. Cadranel J, Wislez M, Antoine M. Primary pulmonary lymphoma. Eur Respir J 2002;20(3):750-762.

2. Marchiori E, Valiante PM, Gutierrez AL, Bodanese L, Souza Júnior AS. [Pulmonary lymphomas: high-resolution computed tomography and anatomopathological correlation]. Radiol Bras 2002;35(1):1-6. Article in Portuguese.

3. Lee KS, Kim Y, Primack SL. Imaging of pulmonary lymphomas. AJR 1997;168(2):339-345. 


\section{Lymphoma ACCOMPanied By a Hydatid Cyst}

4. Sankaranarayanan V, Zeidalski TM, Chitkara RK. A 55-year-old smoker with a persistent right lower lobe infiltrate. Chest 2005; 127(6):2266-2270.

5. Kim JH, Lee SH, Park J, Kim HY, Lee SI, Park JO, et al. Primary pulmonary non-Hodgkin's lymphoma. Jpn J Clin Oncol 2004;34(9): 510-514.

6. Kinsely BL, Mastey LA, Mergo PJ, Voytovich MC, Zander D, Almasri NM, et al. Pulmonary mucosa-associated lymphoid tissue lymphoma: CT and pathologic findings. AJR 1999;172(5):13211326.

7. Mattedi RL, Bernardi Fdel C, Bacchi CE, Siqueira SA, Mauad T. Fatal outcome in bronchus-associated lymphoid tissue lymphoma. J Bras Pneumol 2007;33(4):487-491.
8. Martínez Rivera C, Bonnin Vilaplana M, Simón Adiego C, Palacín Forgué A, Puig Zuza J, Sampablo Lauro I. Primary pulmonary lymphoma presenting as a pulmonary mass with cavitation. Arch Bronconeumol 2004;40(2):94-96.

9. Lazar EB, Whitman GJ, Chew FS. Lymphoma of bronchus associated lymphoid tissue. AJR 1996;167(1):116.

10. Lee IJ, Kim SH, Koo SH, Kim HB, Hwang DH, Lee KS, et al. Bronchus-associated lymphoid tissue (BALT) lymphoma of the lung showing mosaic pattern of inhomogeneous attenuation on thin-section CT: a case report. Korean J Radiol 2000;1(3):159-161.

11. Luce JA. Lymphoma, lymphoproliferative diseases, and other primary malignant tumors. In: Murray JF, Nadel JA, editors. Textbook of respiratory medicine. Philadelphia: WB Saunders; 2000:1453-1468. 